\section{Análise dos padrões espaciais e caracterização dos suicídios no Brasil entre 1990 e 2015}

\author{
Analysis of spatial patterns and characterization \\ of suicides in Brazil from 1990 to 2015
}

\section{Análisis de los patrones espaciales y caracterización \\ de los suicidios en Brasil entre 1990 y 2015}

Danielly Cristina de Andrade Palma 1,2

Emerson Soares dos Santos 3

Eliane Ignotti 2

\section{Resumo}

O objetivo deste estudo foi analisar o padrão espacial das taxas de mortalidade por suicídio no Brasil entre 1990 e 2015. Realizou-se análise espacial das taxas de mortalidade por suicídio dos municípios, em triênios, por meio de inferência bayesiana e análise de clusters, segundo risco de óbito ajustado por sexo e faixa etária. A taxa de mortalidade por suicídio aumentou de 3,5 óbitos em 1990 para 5,3 óbitos/100 mil habitantes em 2015 quando, a cada 64 minutos, uma morte foi registrada. Houve predominância de óbitos masculinos em todos os períodos, e as variáveis idade e raça/cor apresentaram alterações. Alta taxa de mortalidade entre jovens e indígenas foi observada no último triênio. Observamos perda de áreas sem notificação, bem como redução de áreas com taxas baixas de mortalidade em oposição ao aumento de áreas com taxas médias em todas as regiões. As taxas altas, que se concentravam no Sul, se dispersaram para outras regiões. Taxas muito altas surgiram no sul de Mato Grosso do Sul. Clusters de maior verossimilhança em todos os períodos são observados no Sul. As taxas de mortalidade por suicídio aumentaram, com alteração no padrão espacial no período estudado. Apesar da concentração inicial das altas taxas de mortalidade no Sul, houve dispersão dos óbitos para as demais regiões com diferentes magnitudes. Por um lado, houve redução dos óbitos entre idosos no Sul; por outro lado, houve aumento de óbitos no Nordeste, de adultos e pessoas negras no Centro-oeste e entre jovens e indígenas no noroeste do Amazonas.

Suicídio; Conglomerados Espaço-Temporais; Teorema de Bayes

\author{
Correspondência \\ D. C. A. Palma \\ Universidade Federal de Mato Grosso do Sul. \\ Av. Márcio Lima Nantes $s / n$, Estrada do Pantanal, Coxim, MS \\ 79400-000, Brasil. \\ danielly_palma@yahoo.com.br \\ 1 Universidade Federal de Mato Grosso do Sul, Coxim, Brasil. \\ 2 Universidade do Estado de Mato Grosso, Cáceres, Brasil. \\ 3 Universidade Federal do Mato Grosso, Cuiabá, Brasil.
}




\section{Introdução}

O suicídio é considerado um evento complexo e multicausal 1 , e a identificação de áreas de maior risco é uma ferramenta para o planejamento de estratégias mais eficazes para sua prevenção. Segundo a Organização Mundial da Saúde (OMS) 1, em 2012, foram notificadas aproximadamente 804 mil mortes por suicídio no mundo (11,4/100 mil habitantes), das quais mais de $70 \%$ ocorreram em países de baixa e média renda. O número de suicídios entre homens é três vezes maior do que entre mulheres em países desenvolvidos; em países em desenvolvimento, essa relação se reduz pela metade. Mundialmente o suicídio corresponde a 50\% das mortes violentas entre homens e 71\% entre mulheres. Idosos a partir dos setenta anos de idade apresentam maior taxa de mortalidade por suicídio. Porém, em alguns países, as taxas são mais elevadas entre os jovens, principalmente entre 15 e 29 anos, chegando a ser a segunda principal causa de morte nesse grupo etário. Os métodos mais empregados são ingestão de agrotóxicos, enforcamento e uso de arma de fogo 1. As taxas de suicídio (por $100 \mathrm{mil} /$ habitantes) distribuem-se mundialmente em 15,4 na Europa, 13,2 no Sudeste Asiático, 10,2 no Oeste do Pacífico, 9,8 nas Américas, 7,4 na África e 3,9 no Mediterrâneo Oriental 2.

O Brasil é o oitavo país em número absoluto de óbitos por suicídio, ranqueado como a quarta causa de notificação por morte por causas externas no país 1 (Departamento de Informática do SUS. http://tabnet.datasus.gov.br/cgi/tabcgi.exe?sim/cnv/ext10uf.def, acessado em 27/Set/2018). Apesar da obrigatoriedade no preenchimento da causa da morte na Declaração de Óbito (DO), a notificação compulsória do suicídio é relativamente recente no país e passou a ser realizada de fato em 2011. Em 2014, o Ministério da Saúde publicou a Portaria no 1.271/2014 3, que inclui a tentativa de suicídio na lista de agravos de notificação imediata pelo município, levando em consideração a importância da tomada rápida de decisão no encaminhamento e vinculação do paciente aos serviços de atenção psicossocial, prevenindo, assim, uma nova tentativa e possível consumação desse tipo de morte.

Segundo dados publicados no Boletim Epidemiológico do Ministério da Saúde em 2017 4, os óbitos por suicídio no Brasil, no período de 2011 a 2016, aumentaram em ambos os sexos. Independentemente do sexo, as maiores taxas foram observadas nas faixas etárias mais elevadas (a partir dos 70 anos), com até três anos de estudos e na população indígena, principalmente entre os adolescentes de 10 a 19 anos. Taxas mais elevadas foram registradas nos estados do Rio Grande do Sul, Santa Catarina e Mato Grosso do Sul. As informações do Boletim Epidemiológico são de extrema importância para o conhecimento e a discussão sobre a ocorrência de óbitos por suicídio no Brasil, porém não abordam as alterações ocorridas ao longo dos anos, informação que pode colaborar na compreensão da ocorrência do evento no país e contribuir com a elaboração de políticas públicas que auxiliem a redução dos casos.

Este trabalho teve como objetivo analisar o perfil epidemiológico e as mudanças no padrão espacial da taxa de mortalidade por suicídio no Brasil entre 1990 e 2015.

\section{Método}

Estudo ecológico exploratório, com análise espacial das taxas de mortalidade por suicídio dos municípios brasileiros no período de 1990 a 2015. São analisadas a distribuição das taxas bayesianas e a ocorrência de clusters de municípios com alta incidência de óbitos por suicídio.

Para a análise do período de 25 anos, utilizaram-se dados agrupados em três triênios: 1990-1992, 2000-2002 e 2013-2015. Assumiram-se os anos de 1991, 2001 e 2014 como referências para as bases populacionais e territoriais do Instituto Brasileiro de Geografia e Estatística (IBGE) para cada período. O intervalo maior de tempo entre o segundo e o terceiro triênios é decorrente da disponibilidade de malhas territoriais pelo IBGE e do emprego de dados recentes de óbitos por suicídio.

Os dados sobre mortalidade foram obtidos no Sistema de Informações sobre Mortalidade (SIM) do Ministério da Saúde. Para o primeiro triênio, foi utilizada a 9ạ Classificação Internacional de Doenças (CID-9), código E54 (Suicídios e lesões autoinfligidas). Para os demais triênios, foi utilizada a 10a Classificação Internacional de Doenças (CID-10), códigos X60-X69 (Autointoxicação intencional) e X70-X84 (Lesões autoprovocadas voluntariamente). Foram excluídos os óbitos dos municípios que constavam como ignorados: 111 (primeiro triênio), 104 (segundo triênio) e 74 (terceiro triênio). 
Em face do expressivo número de óbitos por causas externas com intenção indeterminada, os óbitos por suicídio foram corrigidos por meio da proporção entre os óbitos por suicídio e o número total de óbitos por causas externas. Assumiu-se que a distribuição das causas de óbitos entre os que ocorreram com intenção indeterminada é semelhante à distribuição das causas por causas definidas.

Esse procedimento 5 foi seguido em todos os períodos de estudo e pode ser resumido pela seguinte equação:

$$
X s=X+M * X /(T-M)
$$

onde Xs é o número corrigido de óbito por suicídio; X é o número de óbitos por suicídio; M é o número de óbitos por intenção indeterminada; e T é o número de óbitos por causas externas.

As taxas de mortalidade para cada município foram estimadas por meio do método de inferência bayesiana, utilizando o Modelo Bayesiano Empírico Local que, para estimar uma taxa para cada município, inclui efeitos espaciais. O método Bayesiano Empírico Local estima uma taxa levando em conta o valor observado em cada município e a média dos valores observados em seus vizinhos adjacentes 6 . Em áreas com populações reduzidas, a confiança na taxa observada diminui 7, mas com o uso dessa técnica, a flutuação aleatória é atenuada, permitindo comparações entre os municípios, mesmo que tenham populações distintas. Foram construídas taxas médias de cada triênio para cada município. Para a matriz de vizinhança, consideraram-se apenas os vizinhos de primeira ordem. Neste trabalho, o uso da inferência bayesiana tem o objetivo de construir mapas de taxas de mortalidade para análise exploratória, categorizados de acordo com Diekstra \& Gulbinat 8 . O cálculo das taxas bayesianas foi realizado com o software TerraView 3.4 (http://www.dpi.inpe.br/terraview).

Para a identificação de clusters de risco, foi realizada a técnica de varredura espacial SCAN, tendo sexo e idade como variáveis de ajuste, com o uso do software SaTScan 9.6 (http: \\www.satscan.org). Essa técnica examina janelas circulares, com raio variável ou não, em que a área delimitada pela janela de análise, intitulada de região z, pode constituir um cluster se o valor encontrado for maior ou menor que o esperado. Para cada janela examinada, testa-se a hipótese nula $\left(\mathrm{H}_{0}\right)$ de ausência de cluster na região de estudo, frente à hipótese alternativa $\left(\mathrm{H}_{1}\right)$, considerando a região $\mathrm{z}$ como um cluster. Nesse caso, assume-se a existência de um risco de óbito por suicídio em comparação com a janela exterior 9.

$\mathrm{Na}$ análise SCAN, para cada um dos triênios, rodou-se a análise puramente espacial por meio do modelo discreto de Poisson, determinando-se os seguintes parâmetros: ausência de sobreposição geográfica, tamanho máximo do cluster igual a 50\% da população sob risco, cluster em formato circular com raio máximo de 500 quilômetros e 999 replicações de Monte Carlo para o teste de significância dos clusters. Para a elaboração dos mapas, foram considerados apenas os clusters estatisticamente significantes para intervalo de 95\% de confiança (IC95\%). A estratificação foi realizada pelo valor da razão de taxas denominada risco relativo (RR) de cada cluster.

Os clusters foram caracterizados segundo o perfil da mortalidade por suicídio, de acordo com as covariáveis sexo, faixa etária e raça/cor, calculando-se o coeficiente de mortalidade por suicídio por $100 \mathrm{mil} /$ habitantes para cada covariável.

O estudo foi aprovado pelo Comitê de Ética em Pesquisa com Seres Humanos da Universidade do Estado de Mato Grosso (UNEMAT), sob o parecer de no 2.602.858.

\section{Resultados}

De 1990 a 2015, foram notificadas no Brasil 205.431 mortes por suicídio, com uma média de 8.217 mortes anuais e 22 diárias. A taxa de mortalidade por suicídio no Brasil aumentou 53\% no período, passando de 3,5 para 5,3 óbitos por 100 mil habitantes.

Até 2001, os municípios não notificadores de óbitos por suicídio estavam concentrados nas regiões Norte e Nordeste. Na comparação do primeiro com o último triênio, foi observada redução e quase o desaparecimento de áreas sem notificação (-92\%). Ocorreu também redução de 11\% das áreas com taxas baixas de mortalidade por suicídio (<5/100 mil habitantes) em oposição ao aumento de $182 \%$ das áreas com taxas médias (5 a 15/100 mil habitantes) em todas as regiões do país. Os municípios com taxas altas (15 a 30/100 mil habitantes) concentrados no sul da Região Sul passam a ser observados no 
segundo triênio, em Mato Grosso do Sul e Mato Grosso, com aumento de 161\%. Em 2014, altas taxas de mortalidade por suicídio foram claramente observadas na região do Alto Solimões, a noroeste do Estado do Amazonas, e em algumas áreas das regiões Sudeste e Norte. As taxas muito altas (> 30/100 mil habitantes) foram observadas no segundo triênio e permanecem no último triênio no sul de Mato Grosso do Sul (Figura 1).

Acerca da formação de clusters (Figura 2), observa-se que, em todos os períodos analisados, o cluster primário é formado por municípios que abrangem os três estados da Região Sul e parte do sul de Mato Grosso do Sul. Nesses locais, a configuração espacial e o RR apresentam alterações entre os períodos

\section{Figura 1}

Distribuição espacial das taxas de mortalidade por suicídio por meio de inferência bayesiana no Brasil nos triênios 1990-1992, 2000-2002 e 2013-2015.

1a) 1991

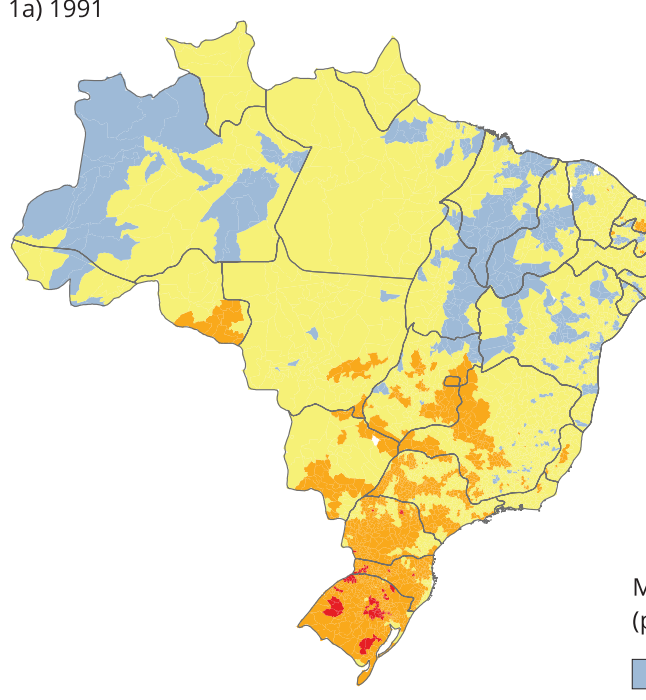

rtalidade por suicídio (por 100.000 habitantes)

0,0

$0,1-5,0$

$5,1-15,0$

1c) 2014

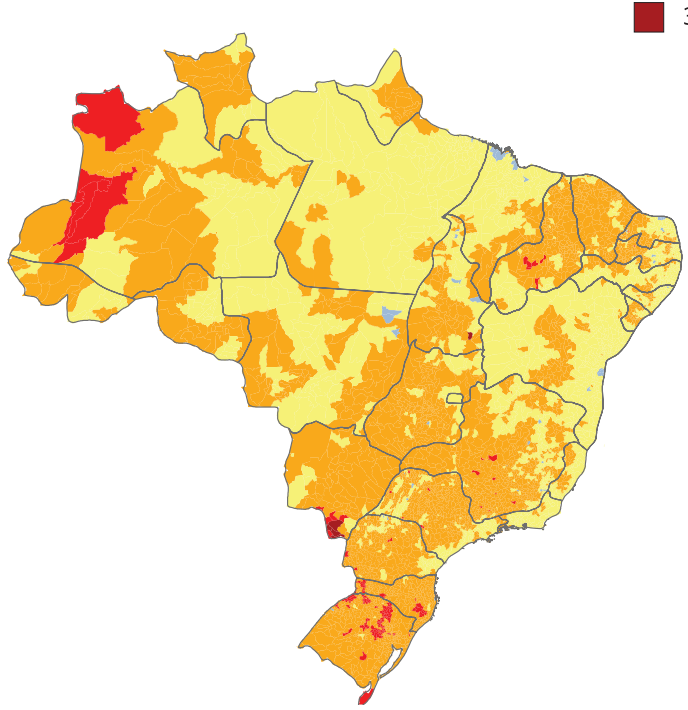

1b) 2001

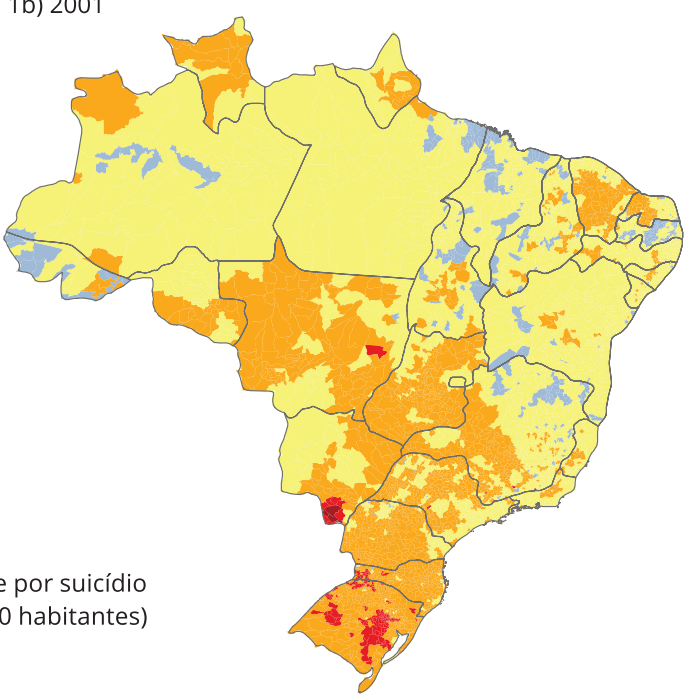

$15,1-30,0$

$30,1-250,0$

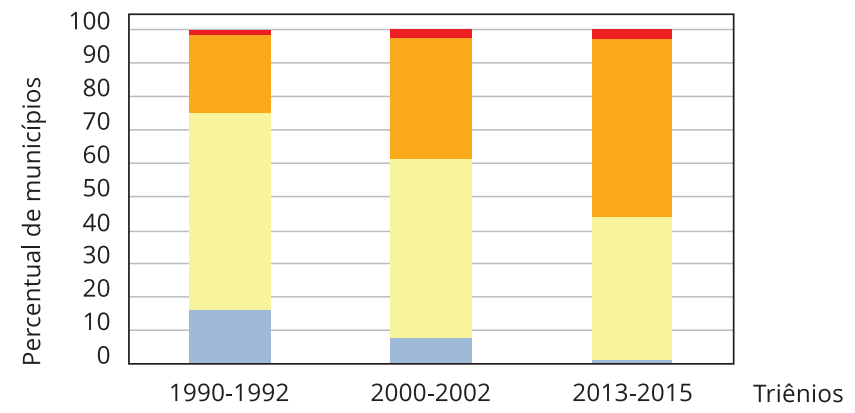




\section{Figura 2}

Distribuição espacial dos clusters espaciais da mortalidade por suicídio no Brasil nos triênios 1990-1992, 2000-2002 e $2013-2015$.

2a) 1991

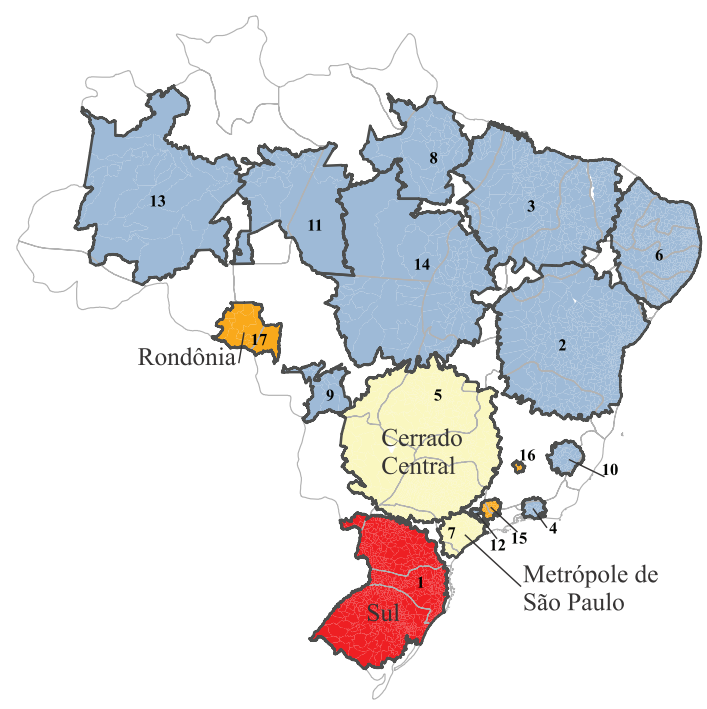

2c) 2014

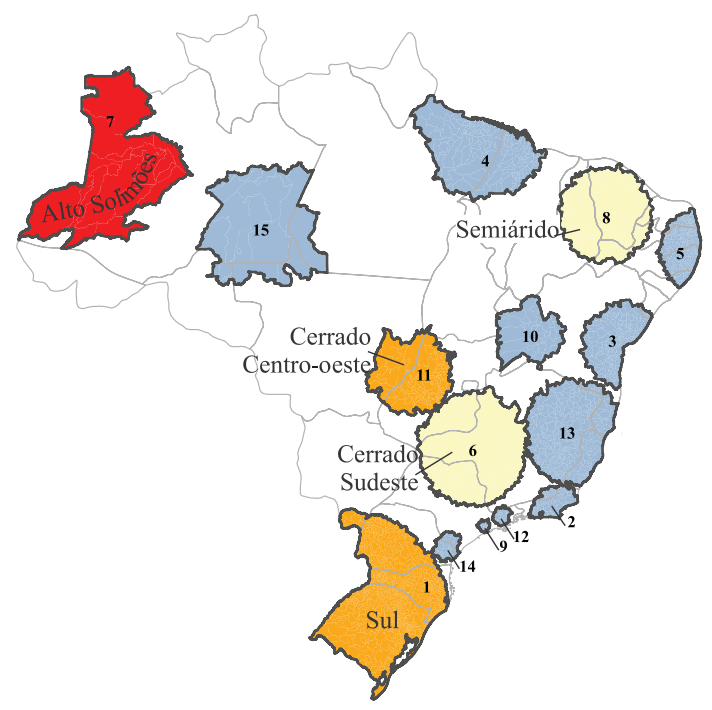

2b) 2001

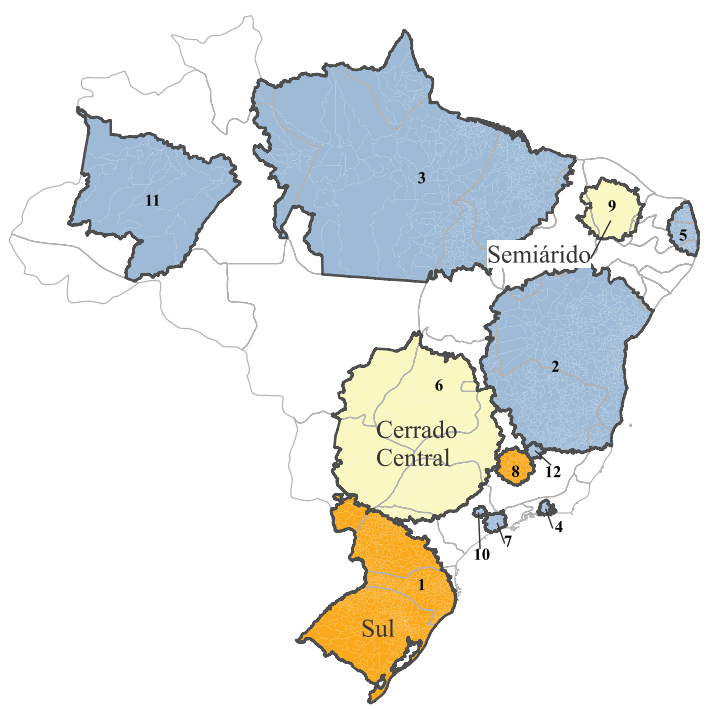

3 clusters

Risco relativo

$0,10-1,00$

$1,1-1,50$

$1,51-2,50$

$2,51-3,60$

$(R R=2,72 ; R R=2,62 ; R R=2,06$, respectivamente), mas mantém-se o dobro da taxa de mortalidade do país. Nos anos 1991 e 2001, foi constatada a formação de um cluster no Cerrado Central, sendo composto de municípios das regiões Centro-oeste, Sudeste e Sul. Em 2014, percebe-se a divisão desse cluster, ocasionando a formação de outros dois com RR diferentes (Cerrado Centro-oeste, $\mathrm{RR}=1,68$; e Cerrado Sudeste, $\mathrm{RR}=1,45$ ).

Em 2001, ocorreu a formação do cluster Semiárido Nordestino $(R R=1,42)$ em uma área onde até então se formavam clusters categorizados como "sem risco" para óbito por suicídio. Esse cluster tem sua 
extensão aumentada em 2014 e RR = 1,39, apresentando redução em comparação ao período anterior. Nesse último período, com a presença de municípios com altas taxas de incidência registradas no Alto Solimões, observa-se a formação de um cluster com maior RR (2,92). A formação de clusters "sem risco" diminuiu consideravelmente em quantidade e, principalmente, em extensão territorial.

As características dos clusters em relação a estatística SCAN e perfil epidemiológico quanto a sexo, idade e raça/cor podem ser observadas nas Tabelas 1, 2 e 3.

Em relação à primeira variável, os óbitos no sexo masculino predominaram em todos os cluster nos três períodos analisados. Quanto a idade, em 1991, os óbitos foram predominantes em indivíduos com idade igual ou superior a 60 anos, com exceção do cluster Rondônia, onde a maioria dos óbitos ocorreu em indivíduos de 25 a 59 anos. No período de análise, foi observada redução na taxa de óbitos por suicídio entre idosos no Sul (19\%) e aumento na mesma faixa etária no Nordeste (35\%), mantendose essa taxa estável na região do Cerrado. Na faixa etária dos 25 a 59 anos, os óbitos mantiveram-se estáveis tanto no Sul como no Nordeste, com aumento de $13 \%$ na região do Cerrado. Com a divisão do cluster Cerrado Central, na sua porção Centro-oeste, a diferença das taxas entre adultos e idosos é maior (2,63/100 mil habitantes contra 0,81/100 mil habitantes na porção Cerrado Sudeste).

Em 2014, chama a atenção o cluster Alto Solimões, que apresenta alta taxa de mortalidade entre jovens e indígenas. Ao considerarmos somente a variável raça/cor, destaca-se a alta taxa de mortalidade entre indígenas em 2001 e 2014, além da mortalidade de negros nos clusters Cerrado Centro-oeste e Semiárido Nordestino em 2014.

\section{Discussão}

As taxas de óbitos por suicídio no Brasil aumentaram no período estudado, com alterações importantes em sua distribuição espacial. Os 205.431 óbitos por suicídio notificados entre 1991 e 2015 ou a taxa de 5,3 óbitos por 100 mil habitantes no último triênio mostra uma média de um suicídio a cada 64 minutos no Brasil.

Diferentes pesquisas realizadas no Brasil descrevem aumento na taxa de óbitos por suicídio. Mello-Santos et al. 10 relataram um aumento de 21\% entre 1980 e 2000 (de 3,3 para 4,0 óbitos/100 mil habitantes). Machado \& Santos 11, por sua vez, destacaram que houve um aumento de $26 \%$ entre 2000 e 2012 (de 4,9 para 6,2 óbitos/100 mil habitantes). Para o ano 2000, os trabalhos citados encontraram valores díspares, 4,0 e 4,9 óbitos/100 mil habitantes respectivamente, o que pode ser explicado pela defasagem de dois a quatro anos na disponibilização de dados consistentes sobre mortalidade. Botega 12 explica que esse lapso de tempo se deve ao cuidado na verificação e na consolidação dos dados, uma vez que, para se notificar um óbito por suicídio, é necessário estabelecer se houve intencionalidade da morte, e nem sempre isso é possível.

As taxas mais elevadas apresentavam-se concentradas principalmente na Região Sul e, com o passar do tempo, abrangeram municípios das regiões Centro-oeste, Norte e Nordeste. Uma suposição para o crescimento dessas taxas nas regiões Norte e Nordeste é a melhora na qualidade das notificações sobre mortalidade ocorrida ao longo dos últimos anos no Brasil 13.

$\mathrm{Na}$ Região Sul do país, estão localizadas áreas com taxas de mortalidade por suicídio mais elevadas e é onde se formam os clusters de maior verossimilhança em todos os períodos analisados. Não há clareza quanto aos motivos das altas taxas na Região Sul, mas devem estar relacionados com uma complexa combinação de condicionantes socioculturais, econômicos e psicobiológicos entre os agricultores gaúchos, população mais afetada 12,14. Relaciona-se esse fato, ainda, aos exigentes padrões de comportamento social presentes em municípios colonizados por imigrantes europeus, principalmente alemães 15 , à forma peculiar da monocultura do tabaco 16 , ao potencial agravo dos pesticidas 14 , à baixa escolaridade, ao endividamento, à defesa da honra em uma cultura patriarcal, à alta incidência de transtornos mentais e à história de suicídio que acompanha o histórico familiar 12 .

É importante lembrar que a formação desses clusters também é influenciada pelos óbitos de indígenas ao sul do Mato Grosso do Sul. Ali habitam os índios da etnia Guarani/Kaiowá, que correspondem a aproximadamente $60 \%$ da população indígena do estado, e apresentam uma taxa de mortalidade por suicídio 22 vezes maior em relação ao restante da população brasileira 17,18 . Como o suicídio é recorrente nessa população, as vivências, desde os primeiros anos de vida com essa situação, assim 
Tabela 1

Caracterização e perfil epidemiológico dos clusters espaciais da mortalidade por suicídio no Brasil entre 1990 e 1992.

\begin{tabular}{|c|c|c|c|c|c|c|c|c|c|c|}
\hline \multirow[t]{2}{*}{ Cluster } & \multirow[t]{2}{*}{ População } & \multirow{2}{*}{$\begin{array}{l}\text { Casos } \\
\text { obser- } \\
\text { vados }\end{array}$} & \multirow{2}{*}{$\begin{array}{l}\text { Casos } \\
\text { espe- } \\
\text { rados }\end{array}$} & \multirow{2}{*}{$\begin{array}{l}\text { Casos } \\
\text { detec- } \\
\text { tados * }\end{array}$} & \multirow{2}{*}{$\begin{array}{c}\text { Obser- } \\
\text { vados/ } \\
\text { Esperados }\end{array}$} & \multirow[t]{2}{*}{$\mathbf{R R}$} & \multicolumn{2}{|c|}{ IC95\% } & \multirow[t]{2}{*}{ RV } & \multirow{2}{*}{$\begin{array}{c}\text { Valor } \\
\text { de } p * *\end{array}$} \\
\hline & & & & & & & $\begin{array}{l}\text { Limite } \\
\text { inferior }\end{array}$ & $\begin{array}{l}\text { Limite } \\
\text { superior }\end{array}$ & & \\
\hline Primário Sul (1) & 16.139 .485 & 3.863 & 1.776 & 7,60 & 2,18 & 2,72 & 2,62 & 2,82 & $1.088,20$ & $<0,001$ \\
\hline 16 & 270.778 & 61 & 30 & 7,20 & 2,06 & 2,15 & 1,67 & 2,76 & 12,68 & 0,031 \\
\hline Rondônia (17) & 362.000 & 70 & 36 & 6,77 & 1,94 & 1,85 & 1,46 & 2,33 & 12,43 & 0,040 \\
\hline 15 & 794.352 & 151 & 91 & 5,80 & 1,66 & 1,82 & 1,55 & 2,13 & 16,72 & $<0,001$ \\
\hline Cerrado Central (5) & 16.484 .748 & 2.365 & 1.800 & 4,60 & 1,31 & 1,43 & 1,37 & 1,50 & 92,85 & $<0,001$ \\
\hline Metrópole de São Paulo (7) & 13.407 .022 & 1.879 & 1.481 & 4,40 & 1,27 & 1,38 & 1,32 & 1,45 & 55,14 & $<0,001$ \\
\hline 6 & 17.368 .510 & 1.231 & 1.723 & 2,50 & 0,71 & 0,65 & 0,61 & 0,69 & 87,21 & $<0,001$ \\
\hline 12 & 1.442 .208 & 79 & 161 & 1,73 & 0,49 & 0,52 & 0,42 & 0,65 & 25,90 & $<0,001$ \\
\hline 14 & 1.577 .061 & 86 & 158 & 1,90 & 0,55 & 0,52 & 0,42 & 0,64 & 19,73 & $<0,001$ \\
\hline 4 & 10.067.709 & 444 & 1.144 & 1,37 & 0,39 & 0,40 & 0,37 & 0,44 & 297,30 & $<0,001$ \\
\hline 10 & 1.418 .667 & 56 & 147 & 1,30 & 0,38 & 0,37 & 0,29 & 0,49 & 37,51 & $<0,001$ \\
\hline 2 & 12.669 .057 & 339 & 1.235 & 0,97 & 0,27 & 0,24 & 0,21 & 0,27 & 486,11 & $<0,001$ \\
\hline 3 & 9.241 .674 & 231 & 877 & 0,93 & 0,26 & 0,23 & 0,20 & 0,26 & 352,72 & $<0,001$ \\
\hline 8 & 1.099 .961 & 22 & 101 & 0,77 & 0,22 & 0,19 & 0,13 & 0,29 & 45,61 & $<0,001$ \\
\hline 11 & 500.350 & 7 & 47 & 0,53 & 0,15 & 0,13 & 0,06 & 0,28 & 27,09 & $<0,001$ \\
\hline 9 & 684.908 & 8 & 69 & 0,40 & 0,12 & 0,11 & 0,06 & 0,22 & 43,66 & $<0,001$ \\
\hline 13 & 438.919 & 5 & 39 & 0,47 & 0,13 & 0,11 & 0,05 & 0,06 & 23,43 & $<0,001$ \\
\hline
\end{tabular}

\begin{tabular}{|c|c|c|c|c|c|c|c|c|c|c|c|}
\hline \multirow[t]{3}{*}{ Cluster } & & \multirow[t]{3}{*}{$\mathbf{R} \mathbf{R}$} & \multicolumn{9}{|c|}{ Taxa média de mortalidade por suicídio *** } \\
\hline & & & \multicolumn{2}{|c|}{ Sexo } & \multicolumn{3}{|c|}{ Idade (anos) } & \multicolumn{4}{|c|}{ Raça \# } \\
\hline & & & Masculino & Feminino & $1-24$ & 25-59 & $\geq 60$ & Branca & Preta & Amarela & Indígena \\
\hline \multirow[t]{7}{*}{ Alto risco } & Primário Sul (1) & 2,72 & 12,17 & 3,83 & 2,89 & 11,74 & 19,85 & - & - & - & - \\
\hline & 16 & 2,15 & 12,41 & 2,69 & 2,97 & 11,21 & 16,48 & - & - & - & - \\
\hline & Rondônia (17) & 1,85 & 7,42 & 5,38 & 3,53 & 11,52 & 4,60 & - & - & - & - \\
\hline & 15 & 1,82 & 9,79 & 2,80 & 2,70 & 9,51 & 10,68 & - & - & - & - \\
\hline & Cerrado Central (5) & 1,43 & 7,29 & 2,29 & 2,35 & 7,21 & 7,72 & - & - & - & - \\
\hline & Metrópole de São & 1,38 & 7,34 & 2,13 & 2,25 & 6,56 & 8,92 & - & - & - & - \\
\hline & Paulo (7) & & & & & & & & & & \\
\hline \multirow[t]{15}{*}{ Sem risco } & 6 & 0,65 & 3,63 & 1,17 & 0,93 & 4,22 & 4,54 & - & - & - & - \\
\hline & 12 & 0,52 & 3,26 & 0,41 & 1,15 & 2,39 & 2,83 & - & - & - & - \\
\hline & 14 & 0,52 & 2,59 & 0,97 & 0,63 & 3,83 & 1,89 & - & - & - & - \\
\hline & 4 & 0,40 & 2,14 & 0,85 & 0,53 & 2,01 & 3,26 & - & - & - & - \\
\hline & 10 & 0,37 & 1,61 & 1,02 & 0,69 & 1,99 & 2,46 & - & - & - & - \\
\hline & 2 & 0,24 & 1,45 & 0,34 & 0,36 & 1,62 & 1,87 & - & - & - & - \\
\hline & 3 & 0,23 & 1,28 & 0,40 & 0,28 & 1,72 & 1,66 & - & - & - & - \\
\hline & 8 & 0,19 & 0,82 & 0,50 & 0,28 & 1,35 & 1,93 & - & - & - & - \\
\hline & 11 & 0,13 & 0,89 & - & 0,31 & 0,87 & - & - & - & - & - \\
\hline & 9 & 0,11 & 0,58 & 0,20 & 0,25 & 0,51 & 1,04 & - & - & - & - \\
\hline & 13 & 0,11 & 0,59 & 0,16 & 0,22 & 0,27 & 4,28 & - & - & - & - \\
\hline & Mínimo & 0,11 & 0,58 & 0,16 & 0,22 & 0,27 & 1,04 & - & - & - & - \\
\hline & Média & 0,87 & 4,43 & 1,48 & 1,31 & 4,62 & 5,53 & - & - & - & - \\
\hline & Mediana & 0,52 & 2,59 & 0,97 & 0,69 & 2,39 & 3,26 & - & - & - & - \\
\hline & Máxima & 2,72 & 12,41 & 5,38 & 3,53 & 11,74 & 19,85 & - & - & - & - \\
\hline
\end{tabular}

IC95\%: intervalo de 95\% de confiança; RR: risco relativo; RV: razão de verossimilhança.

* Taxa média de mortalidade (por 100 mil habitantes) do período;

** Determinado por 999 replicações de Monte Carlo;

*** Por 100 mil habitantes;

\# A variável passou a integrar a Declaração de Óbito somente em 1996. 
Tabela 2

Caracterização e perfil epidemiológico dos clusters espaciais da mortalidade por suicídio no Brasil entre 2000 e 2002.

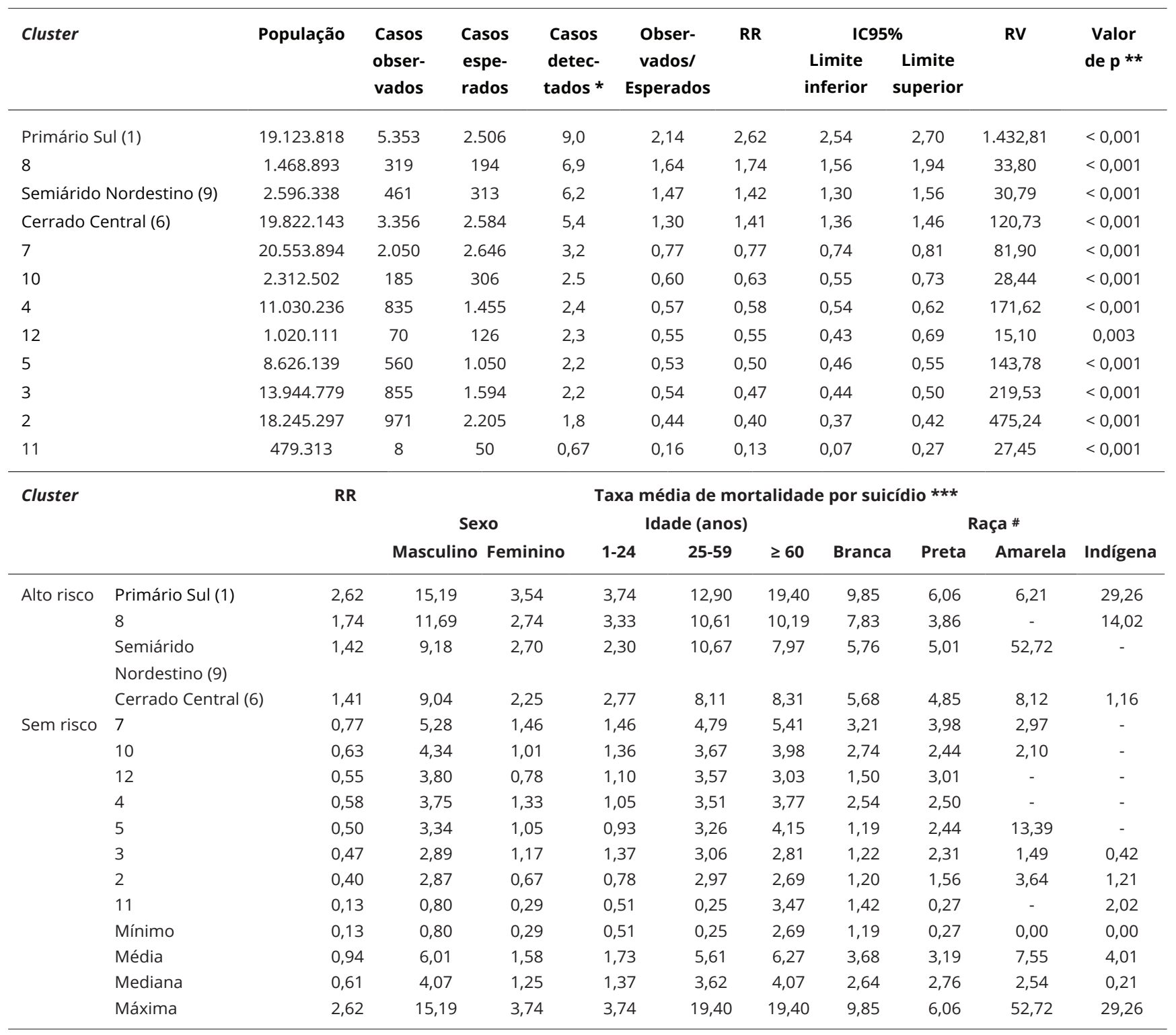

IC95\%: intervalo de 95\% de confiança; RR: risco relativo; RV: razão de verossimilhança.

* Taxa média de mortalidade (por 100 mil habitantes) do período;

** Determinado por 999 replicações de Monte Carlo;

*** Por 100 mil habitantes. 
Tabela 3

Caracterização e perfil epidemiológico dos clusters espaciais da mortalidade por suicídio no Brasil entre 2013 e 2015.

\begin{tabular}{|c|c|c|c|c|c|c|c|c|c|c|c|c|c|c|}
\hline \multirow[t]{2}{*}{ Cluster } & & \multirow{2}{*}{\multicolumn{2}{|c|}{ População }} & \multirow{2}{*}{$\begin{array}{l}\text { Casos } \\
\text { obser- } \\
\text { vados }\end{array}$} & \multirow{2}{*}{\multicolumn{2}{|c|}{$\begin{array}{l}\text { Casos } \\
\text { espe- } \\
\text { rados }\end{array}$}} & \multirow{2}{*}{$\begin{array}{c}\text { Casos } \\
\text { detec- } \\
\text { tados * }\end{array}$} & \multirow{2}{*}{\multicolumn{2}{|c|}{$\begin{array}{c}\text { Obser- } \\
\text { vados/ } \\
\text { * Esperados }\end{array}$}} & \multirow[t]{2}{*}{ RR } & \multicolumn{2}{|c|}{ IC95\% } & \multirow[t]{2}{*}{ RV } & \multirow{2}{*}{$\begin{array}{l}\text { Valor } \\
\text { de } p * *\end{array}$} \\
\hline & & & & & & & & & & & $\begin{array}{l}\text { Limite } \\
\text { inferior }\end{array}$ & $\begin{array}{l}\text { Limite } \\
\text { superior }\end{array}$ & & \\
\hline \multicolumn{2}{|c|}{ Primário Sul (1) } & \multicolumn{2}{|c|}{19.388 .256} & 5.753 & \multicolumn{2}{|c|}{3.205} & \multicolumn{2}{|c|}{9,53} & 1,80 & 2,06 & 2,00 & 2,11 & 932,88 & $<0,001$ \\
\hline \multicolumn{2}{|c|}{ Alto Solimões (7) } & \multicolumn{2}{|c|}{482.407} & 223 & \multicolumn{2}{|c|}{64} & \multicolumn{2}{|c|}{18,5} & & 2,92 & 2,56 & 3,33 & 120,37 & $<0,001$ \\
\hline Cerrado Ce & entro-oeste (11) & 1.415. & & 375 & & 35 & 8,5 & & & 1,68 & 1,51 & 1,85 & 35,51 & $<0,001$ \\
\hline Cerrado Su & ıdeste (6) & 14.743 & & 3.298 & & 461 & 7,1 & & & 1,45 & 1,40 & 1,51 & 140,48 & $<0,001$ \\
\hline Semiárido I & Nordestino (8) & 8.751. & & 1.896 & & 335 & 7,5 & & & 1,39 & 1,32 & 1,45 & 109,35 & $<0,001$ \\
\hline 13 & & 10.006 & & 1.304 & & 609 & 4,3 & & & 0,81 & 0,77 & 0,86 & 32,34 & $<0,001$ \\
\hline 9 & & 19.800 & & 2.492 & & 196 & 4,1 & & & 0,77 & 0,74 & 0,81 & 92,30 & $<0,001$ \\
\hline 14 & & 3.412 . & & 400 & & 47 & 3,9 & & & 0,73 & 0,67 & 0,81 & 22,16 & $<0,001$ \\
\hline 5 & & 14.287 & & 1.440 & & 188 & 3,5 & & & 0,62 & 0,59 & 0,65 & 154,72 & $<0,001$ \\
\hline 12 & & 1.929. & & 182 & & 18 & 3,0 & & & 0,59 & 0,51 & 0,68 & 34,72 & $<0,001$ \\
\hline 2 & & 15.559 & & 1.342 & & 542 & $2, \varepsilon$ & & & 0,52 & 0,50 & 0,55 & 366,59 & $<0,001$ \\
\hline 3 & & 9.490. & & 743 & & 490 & 2,6 & & & 0,48 & 0,45 & 0,52 & 238,93 & $<0,001$ \\
\hline 4 & & 8.829. & & 685 & & 296 & $2, \varepsilon$ & & & 0,48 & 0,44 & 0,51 & 180,40 & $<0,001$ \\
\hline 5 & & 582.2 & & 41 & & 34 & 2, & & & 0,44 & 0,33 & 0,60 & 13,76 & 0,016 \\
\hline 10 & & 974.1 & & 42 & & 52 & 1,5 & & & 0,27 & 0,20 & 0,37 & 56,03 & $<0,001$ \\
\hline Cluster & & & $\mathbf{R R}$ & & & & & Tax & ia de $\mathrm{m}$ & nortalidad & de por suic & zídio *** & & \\
\hline & & & & & Se & xo & & & de (ano & & & & aça \# & \\
\hline & & & & Masc & & Femi & ino & $1-24$ & $25-59$ & $\geq 60$ & Branca & Preta & Amarela & Indígena \\
\hline Alto risco & Primário Sul (1) & & 2,06 & 15 & & 4,1 & & 3,77 & 12,57 & 16,73 & 10,68 & 5,68 & 2,26 & 31,07 \\
\hline & Alto Solimões (7) & & 2,92 & 23 & & 7,1 & & 15,66 & 16,82 & 2,76 & 2,25 & 7,94 & - & 43,14 \\
\hline & $\begin{array}{l}\text { Cerrado Centro- } \\
\text {-oeste (11) }\end{array}$ & & 1,68 & 13 & & 3,8 & & 3,97 & 11,37 & 14,00 & 7,81 & 9,60 & - & 1,80 \\
\hline & Cerrado Sudeste (6) & & 1,45 & 11 & & 3,2 & & 2,80 & 10,37 & 9,56 & 7,66 & 6,84 & 1,36 & - \\
\hline Sem risco & $\begin{array}{l}\text { Semiárido Nordestin } \\
\text { (8) }\end{array}$ & & 1,39 & 11 & & 2,8 & & 2,87 & 10,36 & 12,21 & 3,81 & 7,55 & 1,60 & 1,49 \\
\hline & 13 & & 0,81 & 6 , & & 1,9 & & 1,56 & 6,53 & 5,05 & 3,55 & 4,55 & 1,13 & - \\
\hline & 9 & & 0,77 & 6 , & & 1,8 & & 1,65 & 6,05 & 4,25 & 4,54 & 3,75 & 3,12 & 1,51 \\
\hline & 14 & & 0,73 & 6, & & 1,8 & & 1,89 & 5,42 & 4,15 & 4,70 & 1,47 & 2,97 & - \\
\hline & 5 & & 0,62 & 5, & & 1,4 & & 1,49 & 4,48 & 5,93 & 1,28 & 4,50 & 0,23 & 2,07 \\
\hline & 12 & & 0,59 & 5 & & 1,1 & & 0,83 & 4,72 & 3,73 & 3,33 & 2,19 & 3,56 & - \\
\hline & 2 & & 0,52 & 4, & & 1,4 & & 1,14 & 3,85 & 3,91 & 3,36 & 2,46 & - & - \\
\hline & 3 & & 0,48 & 4, & & 0,9 & & 0,87 & 3,58 & 4,93 & 2,09 & 2,68 & 0,88 & - \\
\hline & 4 & & 0,48 & 4 & & 1,0 & & 1,57 & 3,55 & 3,55 & 1,42 & 2,92 & 0,44 & 1,99 \\
\hline & 5 & & 0,44 & 3, & & 1,0 & & 1,36 & 3,76 & 1,97 & 1,98 & 2,36 & - & 4,02 \\
\hline & 10 & & 0,27 & 2, & & 0,5 & & 0,60 & 2,05 & 2,50 & 0,63 & 1,77 & - & - \\
\hline & Mínimo & & 0,27 & 2, & & 0,5 & & 0,60 & 2,05 & 1,97 & 0,63 & 1,47 & 0,00 & 0,00 \\
\hline & Média & & 1,01 & 8, & & 2,3 & & 2,80 & 7,03 & 6,35 & 3,94 & 4,42 & 1,17 & 5,81 \\
\hline & Mediana & & 0,73 & 6, & & 1,8 & & 1,57 & 5,42 & 4,25 & 3,36 & 3,75 & 0,88 & 1,49 \\
\hline & Máxima & & 2,92 & 23 & & 7,1 & & 15,66 & 16,82 & 16,73 & 10,68 & 9,60 & 3,56 & 43,14 \\
\hline
\end{tabular}

IC95\%: intervalo de 95\% de confiança; RR: risco relativo; RV: razão de verossimilhança.

* Taxa média de mortalidade (por 100 mil habitantes) do período;

** Determinado por 999 replicações de Monte Carlo;

*** Por 100 mil habitantes. 
como o contexto social e cultural, podem influenciar na alta taxa de mortalidade por suicídio desse povo. A desagregação cultural e o desarranjo interno do grupo, com a contribuição da não passagem dos jovens pelo rito de iniciação na transição para a fase adulta, também são fatores que contribuem para o suicídio dos Guarani/Kaiowá 17.

Uma outra área importante para mortalidade de indígenas é o cluster Alto Solimões. Não há como afirmar se, em períodos anteriores, nessa área não ocorria suicídio de indígenas ou se não eram notificados. O Conselho Indigenista Missionário (CIMI) 18 relata que a falta de perspectiva para viver em paz nas terras demarcadas e a insegurança quanto à garantia ou manutenção dos direitos estabelecidos constitucionalmente são determinantes para o suicídio indígena. Evitamento da desonra, reações de luto, fuga da escravidão, frustração amorosa, indefinição do status social em decorrência da abolição dos ritos de passagem da infância para a adolescência, dificuldade de acesso às universidades e ao mercado de trabalho e elevado consumo de álcool são fatores que influenciam o suicídio de indígenas 12 .

O suicídio apresenta diferenças importantes entre homens e mulheres, uma vez que se adotam comportamentos autodestrutivos congruentes com as peculiaridades de cada gênero. Mundialmente os homens cometem suicídio de três a quatro vezes mais que as mulheres 1 , resultado semelhante ao encontrado neste estudo para o Brasil.

Há diferentes explicações nas tentativas de elucidar as causas das altas taxas de suicídio entre os homens: o impacto de questões socioeconômicas associadas ao desempenho de papéis sociais que são peculiares aos homens ${ }^{19}$, o desempenho escolar e a capacidade cognitiva geral 20 , a resistência em buscar ajuda psiquiátrica 21, o efeito do divórcio e da alienação parental, a função da testosterona e a maior prevalência de alcoolismo 12 .

Diferenças importantes também são observadas entre as faixas etárias, como a redução na taxa de óbitos por suicídio entre idosos no Sul e o aumento no Nordeste. Estudo realizado por Santos et al. 22, no período de 2000 a 2014, também relata aumento das taxas de mortalidade por suicídio de idosos na Região Nordeste, porém apenas para o sexo masculino.

O suicídio de idosos é considerado pela OMS como um dos mais graves problemas de saúde pública, uma vez que o risco aumenta com a idade e, ao mesmo tempo, essa é a população em maior crescimento no Brasil 23. Estudos indicam que doenças graves e degenerativas, dependência física, distúrbios e sofrimentos mentais, depressão severa, abandono 24, busca por métodos mais letais, maior grau de intencionalidade suicida, menor resistência física e maior propensão a doenças que podem complicar o restabelecimento após uma tentativa de suicídio 12 são alguns fatores predisponentes que aumentam a letalidade do suicídio em idosos.

A alta taxa de mortalidade entre jovens no último período de estudo no cluster Alto Solimões notoriamente está relacionada à alta taxa de mortalidade da raça/cor indígena. O suicídio entre crianças e jovens indígenas no Brasil é visto como uma pandemia e está inserido em um contexto de discriminação, marginalização, colonização traumática e perdas de formas tradicionais de vida que conduzem a reações autodestrutivas do ponto de vista ocidental 25 .

A variável raça/cor teve como limitação a não disponibilidade do dado para o primeiro triênio. A variável foi introduzida na DO a partir de 1996. Em seus primeiros anos de vigência, o preenchimento foi baixo, mas, com o passar do tempo, a completude vem sendo melhorada 26.

Apesar da observação da taxa de óbitos por suicídio para a raça/cor negra ser mais elevada em alguns clusters, não há estudos epidemiológicos específicos sobre o suicídio dessa população no Brasil. Tavares 27 define tal fato como racismo estrutural e institucional, uma vez que essas pessoas correspondem a aproximadamente $54 \%$ da população brasileira, alcançando representatividade numérica no universo estudado, e assim como os indivíduos das demais raças, também estão expostos aos principais fatores predisponentes individuais e coletivos do suicídio. Para Aguiar 28, o suicídio dessa população sofre forte carga das questões sociais, principalmente entre os jovens, que têm maior dificuldade para alcançar o referencial comum criado por uma sociedade que desvaloriza por completo a cidadania afro-brasileira.

A alta taxa de óbitos por suicídio no cluster Semiárido Nordestino em 2001 para a raça/cor amarela, justifica-se pela forma como essa taxa é calculada, uma vez que é relativizada pela população, além de ser pequena dentro do cluster.

A principal limitação para estudos com dados secundários de óbitos por suicídio é a possível subnotificação de mortes com intenção indeterminada ou de causa mal definida que mascara essa 
realidade, uma vez que apenas o modo da morte é registrado, e não a intencionalidade. Fatores que perpassam questões religiosas, sociais, culturais, políticas, econômicas, entre outras influenciam a ocorrência do fato 14 .

Dado o exposto, constatamos que a taxa de óbitos por suicídio no Brasil aumentou no período de 1990 a 2015 e teve seu padrão espacial alterado. A Região Sul apresentou altas taxas em todos os períodos, porém os óbitos deixaram de se concentrar nessa região, passando a atingir todas as demais regiões do país, com diferentes taxas. Apesar de, aparentemente, o risco relativo de óbito por suicídio de alguns clusters ter diminuído ao longo do tempo, é preciso enfatizar que cada mapa é relativizado por si mesmo. Assim, cada cluster foi calculado com a taxa de cada período, e essa taxa aumentou no decorrer do período analisado.

Foram observadas diferenças nos perfis das vítimas de óbitos por suicídio nos diferentes espaços brasileiros. Enquanto no Sul houve redução dos óbitos entre idosos, o inverso foi verificado para o Nordeste. Na região central, que apresentava certa homogeneidade entre as taxas, no último triênio houve diferenciação, com aumento dos óbitos de adultos e de pessoas da raça/cor negra na área de Cerrado do Centro-oeste. Na Amazônia, o cluster Alto Solimões retratou alto índice de óbitos entre jovens indígenas.

\section{Colaboradores}

D. C. A. Palma contribuiu com a concepção e planejamento do estudo, coleta de dados, análise e interpretação dos dados, redação e revisão crítica do manuscrito. E. S. Santos e E. Ignotti contribuiu com a concepção e planejamento do estudo, análise e interpretação dos dados, redação e revisão crítica do manuscrito.

\section{Informações adicionais}

ORCID: Danielly Cristina de Andrade Palma (0000-0002-1128-7874); Emerson Soares dos Santos (0000-0003-2709-5591); Eliane Ignotti (00000002-9743-1856).

\section{Agradecimentos}

O artigo faz parte da tese de doutorado da primeira autora, no Programa de Pós-graduação em Ciências Ambientais da Universidade do Estado de Mato Grosso. Não houve financiamento de agências de fomento.

\section{Referências}

1. World Health Organization. Preventing suicide: a global imperative. https://www.who. int/mental_health/suicide-prevention/world_ report_2014/en/ (acessado em 26/Set/2018).

2. World Health Organization. Suicide rate per 100,000 population by WHO region, 2016. https://www.who.int/mental_health/suicideprevention/per_100.000_population_2016. JPG?ua=1 (acessado em 26/Set/2018).

3. Ministério da Saúde. Portaria no 1.271 , de 6 de junho de 2014. Define a Lista Nacional de Notificação Compulsória de doenças, agravos e eventos de saúde pública nos serviços de saúde públicos e privados em todo território nacional. Diário Oficial da União 2014; 9 jun.

4. Ministério da Saúde. Perfil epidemiológico das tentativas e óbitos por suicídio no Brasil e a rede de atenção à saúde. Boletim Epidemiológico 2017; 48(30).

5. Nunes KVR, Neves SMAS, Ignotti E. Mortality due to diseases of the circulatory system among the elderly populations in Brazilian Amazon: temporal and spatial analysis. Rev Bras Epidemiol 2013; 16:838-48.

6. Souza WV, Carvalho MS, Cruz OG, Ragoni V. Análise espacial de dados de áreas. In: Santos SM, Souza WV, organizadores. Introdução à estatística espacial para a saúde pública. Brasília: Ministério da Saúde; 2007. p. 61-81.

7. Santos SM, Souza WV, organizadores. Introdução à estatística espacial para saúde pública. Brasília: Ministério da Saúde; 2007.

8. Diekstra RF, Gulbinat W. The epidemiology of suicidal behaviour: a review of three continentes. World Health Stat Q 1993; 46:52-68.

9. Kulldorff M, Nagarwalla N. Spatial disease cluster: detection and inference. Stat Med 1995; 14:799-810. 
10. Mello-Santos C, Bertolote JM, Wang YP. Epidemiology os suicide in Brazil (1980-2000): characterization of age and gender rates of suicide. Rev Bras Psiquiatr 2005; 27:131-4.

11. Machado DB, Santos DN. Suicídio no Brasil, 2001 a 2012. J Bras Psiquiatr 2015; 64:45-54.

12. Botega NJ. Crise suicida: avaliação e manejo. Porto Alegre: Artmed; 2015.

13. Cunha CC, Teixeira R, França E. Avaliação da investigação de óbitos por causas mal definidas no Brasil em 2010. Epidemiol Serv Saúde 2017; 26:19-30.

14. Lovisi GM, Santos SA, Legay L, Abelha L, Valencia E. Análise epidemiológica do suicídio no Brasil entre 1980 e 2006. Rev Bras Psiquiatr 2009; 31 Suppl II:S86-93

15. Fraga WS. Determinantes socioeconômicos no Brasil e no Rio Grande do Sul no século XXI [Dissertação de Mestrado]. São Leopoldo: Universidade do Vale do Rio dos Sinos; 2014.

16. Meneghel SN, Moura R. Suicídio, cultura e trabalho em municípios de colonização alemã do Brasil. Interface (Botucatu) 2018; 22:113546.

17. Grubits S, Freire HBG, Noriega JAV. Suicídio de jovens Guarani/Kaiowá de Mato Grosso do Sul, Brasil. Psicol Ciênc Prof 2011; 31:504-17.

18. Conselho Indigenista Missionário. Violência contra os povos indígenas no Brasil - dados de 2016. https://cimi.org.br/pub/relato rio/Relatorio-violencia-contra-povos-indige nas_2016-Cimi.pdf (acessado em 17/Nov/ 2018).

19. Möller-Leimkühler AM. The gender gap in suicide and premature death or: why are mens o vulnerable? Eur Arch Psychiatry Clin Neurosci $2003 ; 253: 1-8$.

20. Sörberg WA, Zeebari Z, Lager A, Gunnell D, Allebeck P, Falkstedt D. Suicide attempt predicted by academic performance and childhood IQ: a cohort of 26000 children. Acta Psychiatr Scand 2018; 137:277-86.
21. Rutz W, Rihmer Z. Suicidality in men - practical issues, challenges, solutions. J Mens Health Gend 2007; 4:393-401.

22. Santos EGO, Oliveira YOMC, Azevedo UN, Nunes ADS, Amador AE, Barbosa IR. Análise espaço-temporal da mortalidade por suicídio em idosos no Brasil. Rev Bras Geriatr Gerontol 2017; 20:854-65.

23. Krug EG, Dahlberg LL, Mercy JA, Zwi AB, Lozano R, editores. Relatório mundial sobre violência e saúde. https://www.opas.org.br/ wp-content/uploads/2015/09/relatorio-mun dial-violencia-saude.pdf (acessado em 20/ Nov/2018).

24. Minayo MCS, Cavalcante FG. Tentativas de suicídio entre pessoas idosas: revisão de literatura (2002/2013). Ciênc Saúde Colet 2015; 20:1751-62

25. Santana R. "Uma verdadeira situação pandêmica de suicídio de jovens indígenas", diz estudo da Flacso Brasil. Conselho Indigenista Missionário - Notícias 2016; 30 jun. https://cimi. org.br/2016/06/38594/.

26. Macente LB, Zandonade E. Avaliação da completude do Sistema de Informação sobre Mortalidade por suicídio na região Sudeste, Brasil, no período de 1996 a 2007. J Bras Psiquiatr 2010; 59:173-81.

27. Tavares JSC. Suicídio na população negra brasileira: nota sobre mortes invisibilizadas. Revista Brasileira de Psicologia 2017; 4:73-5.

28. Aguiar GO. O suicídio entre jovens negros na perspectiva durkheimiana. Identidade! 2012; 17:91-108. 


\section{Abstract}

This study aimed to analyze the spatial pattern in mortality rates from suicide in Brazil from 1990 to 2015. A spatial analysis was performed for the mortality rates from suicide in municipalities (counties) in three-year periods using Bayesian inference and clusters according to risk of death adjusted by sex and age bracket. The mortality rate from suicide increased from 3.5 deaths in 1990 to 5.3 deaths $/ 100,000$ inhabitants in 2015, when there was one death from suicide every 64 minutes. There was a predominance of suicide deaths in males in all the periods, while the variables age and race/color showed some changes. High suicide mortality rates among youth and indigenous people were seen in the most recent three-year period. We observed a loss of areas without suicide reporting, a reduction in areas with low suicide mortality rates, as opposed to an increase in areas with medium rates in all the regions of Brazil. The high rates, previously concentrated in the South of Brazil, had expanded to other regions of the country. Very high rates emerged in southern Mato Grosso do Sul state. Clusters with higher likelihood in all the periods were observed in the South. Suicide mortality rates increased, with changes in the spatial during the 25-year period. Despite the initial concentration of high suicide mortality rates in the South, the deaths spread to other regions of the country with different magnitudes. There was a reduction in suicide deaths among the elderly in the South and an increase in the Northeast, and an increase in deaths in adults and black Brazilians in the Central and among youth and indigenous persons in northwestern Amazonas state.

Suicide; Space-Time Clustering; Bayes Theorem

\section{Resumen}

El objetivo de este estudio fue analizar el patrón espacial de la tasa de mortalidad por suicidio en Brasil entre 1990 y 2015. Se realizó un análisis espacial de las tasas de mortalidad por suicidio en municipios durante trienios, mediante inferencia bayesiana y análisis de clústeres, según el riesgo de fallecimiento, ajustado por sexo y franja de edad. La tasa de mortalidad por suicidio aumentó de 3,5 óbitos en 1990 a 5,3 óbitos/100 mil habitantes en 2015, cuando cada 64 minutos se registró una muerte. Hubo predominancia de fallecimientos masculinos en todos los períodos, mientras que las variables edad y raza/color presentaron alteraciones. Una alta tasa de mortalidad entre jóvenes e indígenas se observó en el último trienio. Observamos pérdida de áreas sin notificación, reducción de áreas con tasas bajas de mortalidad, frente al aumento de áreas con tasas medias en todas las regiones. Las tasas altas, que se concentraban en el Sur, se dispersaron hacia otras regiones. Aparecieron tasas muy altas en el sur de Mato Grosso do Sul. Los clústeres con mayor verosimilitud durante todos los periodos se observan en el Sur. Las tasas de mortalidad por suicidio aumentaron, con alteración en el patrón espacial durante el periodo estudiado. A pesar de la concentración inicial de las altas tasas de mortalidad en el Sur, hubo una dispersión de los fallecimientos hacia las demás regiones con diferentes magnitudes. Hubo una reducción de los óbitos entre ancianos en el Sur y un aumento en el Nordeste, así como un aumento de óbitos de adultos y personas negras en el Centrooeste, $y$ entre jóvenes e indígenas en el noroeste del Amazonas.

Suicidio; Agrupamiento Espacio-Temporal; Teorema de Bayes
Recebido em 23/Mai/2019

Versão final reapresentada em 23/Ago/2019

Aprovado em 27/Set/2019 